\title{
Enfoque inicial del paciente con hemorragia alveolar difusa sin vasculitis pulmonar
}

\section{Initial workup of the patient with diffuse alveolar hemorrhage without pul- monary vasculitis}

Azucena Martínez Caballero(1); Andrea Córdoba Guzmán(1); Robin Alonso Rada Escobar, MD (2).

\section{RESUMEN}

La hemorragia alveolar difusa es una emergencia médica de instauración aguda, cuya aparición es secundaria a procesos fisiopatológicos dentro de la historia natural de un grupo de enfermedades que pueden cursar o no con vasculitis pulmonar. Tanto su reconocimiento como su diagnóstico oportunos permitirán establecer un tratamiento agresivo inmediato, evitar posibles complicaciones e incluso disminuir la alta tasa de mortalidad que engloba esta patología en la actualidad. En esta revisión se realiza un enfoque de cada una de las enfermedades asociadas a la hemorragia alveolar difusa sin vasculitis pulmonar, con especial énfasis en la fisiopatología.

Palabras clave: hemorragia alveolar difusa, hemosiderosis pulmonar idiopática, síndrome de Goodpasture, penicilamina, estenosis mitral, enfermedad veno-oclusiva, linfangioleiomiomatosis/esclerosis tuberosa.

\section{ABSTRACT}

Diffuse alveolar hemorrhage is a rapidly evolving medical emergency, whose appearance is secondary to pathophysiological processes within the natural history of a group of diseases that can present with pulmonary vasculitis or without this condition. Timely recognition and diagnosis enable immediate aggressive treatment, avoiding possible complications, and even reducing the current mortality rate of this condition. This review focuses on the diseases associated with diffuse alveolar hemorrhage without pulmonary vasculitis, laying emphasis on the pathophysiology.

Keywords: diffuse alveolar hemorrhage, idiopathic pulmonary hemosiderosis, Goodpasture syndrome, penicillamine, mitral stenosis, venous occlusive disease, lymphangioleiomiomatosis/tuberous sclerosis.

\section{INTRODUCCIÓN}

La hemorragia alveolar difusa es un síndrome clínico de instauración rápida y progresiva, habitualmente de mal pronóstico debido a su alta morbi-mortalidad. Requiere atención médica inmediata ya que compromete la función respiratoria, y precisa un manejo agresivo en la unidad de cuidados intensivos en más de $90 \%$ de los casos (1).

\section{CONSIDERACIONES GENERALES}

\section{Presentación clínica}

Este síndrome se caracteriza por injuria de la membrana alvéolo-capilar secundario a múltiples procesos fisiopatológicos pertenecientes a la historia natural de varias enfermedades, que como complicación presen-

(1) Estudiantes de Medicina, Universidad Militar - Nueva Granada Hospital Militar Central. Bogotá, Colombia.

(2) Internista-Neumólogo, Jefe del Servicio de Medicina Interna, Hospital Militar Central. Coordinador Académico de Medicina Interna, Universidad Militar Nueva Granada. Bogotá, Colombia.

Correspondencia: azucenaazm@hotmail.com

Recibido: 5 de Marzo de 2012. Aceptado: 22 de Marzo de 2012. 
tan hemorragia alveolar difusa (2). Clínicamente se define por la presencia de hemoptisis (aunque puede no estar en un tercio de los casos, pese a que haya hemorragia alveolar activa), anemia usualmente ferropénica, insuficiencia respiratoria aguda e infiltrados pulmonares difusos en la radiografía de tórax (3).

\section{Hallazgos radiológicos}

La radiografía de tórax revela infiltrados alveolares irregulares, difusos, que pueden iniciar en un patrón focal, unilateral y se vuelven más difusos con el tiempo. Los pacientes que tienen o recurren con hemorragia alveolar aguda pueden desarrollar un patrón alveolar superpuesto sobre cambios intersticiales crónicos. La identificación de las líneas B de Kerley debe dar lugar a una evaluación de la presencia o ausencia de estenosis mitral o pulmonar, o enfermedad veno-oclusiva como etiología de la hemorragia alveolar (4).

\section{Evaluación por laboratorio}

El parámetro de laboratorio que generalmente se ve afectado es el cuadro hemático; se encuentra en la hemoglobina y el hematocrito que representa el grado de anemia usualmente ferropénica. Aquellos pacientes con la forma aguda pueden tener cambios en el hematocrito desde el inicio; otros laboratorios que deben solicitarse son: niveles de creatinina sérica y parcial de orina (orienta a la posibilidad de un síndrome renal - pulmonar) (5). Otras pruebas útiles son: estudio serológico, anticuerpos antimembrana basal, anticuerpos antineutrófilos, marcadores de enfermedad vascular del colágeno, entre otras (6).

\section{Enfoque diagnóstico}

Inicialmente los esfuerzos irán encaminados a una reanimación agresiva; posteriormente el diagnóstico se centrará en una evaluación clínica detallada y en establecer los factores de riesgo para determinadas patologías que como complicación puedan presentar hemorragia alveolar difusa para aproximarse a la etiología más probable (7).

Es importante indagar acerca de síntomas asociados, ocupación del paciente, antecedentes médicos (enfermedades autoinmunes, valvulares, etc.) y farmacológicos, consumo de sustancias ilícitas, entre otros. Con base en lo anterior, junto con los hallazgos del examen físico y las ayudas paraclínicas, podría determinarse si la enfermedad tiene una etiología que enmarque un cuadro con o sin vasculitis pulmonar (8).

\section{ETIOLOGÍAS ESPECÍFICAS}

A continuación se hará una revisión de las etiologías específicas correspondientes a enfermedades que cursan con hemorragia alveolar sin vasculitis pulmonar, con énfasis en la fisiopatología.

\section{Hemosiderosis pulmonar idiopática}

\section{Definición y epidemiología}

La hemosiderosis pulmonar idiopática, también denominada síndrome de Ceelen, fue descrita por primera vez por Virchow en 1864 en un adulto. Es una enfermedad poco frecuente, de etiología desconocida, cuyo diagnóstico se da por exclusión cuando se han descartado causas primarias y secundarias de hemorragia alveolar difusa; se caracteriza por el depósito anormal de hemosiderina, proveniente de la conversión de hierro de la hemoglobina por los macrófagos alveolares (9).

Se produce comúnmente en pacientes pediátricos o adultos jóvenes en edades entre los 16 a 30 años, en este último caso corresponde a $10 \%$ a $20 \%$ de los casos (10), siendo más frecuente en hombres con una relación 3:1 respecto a las mujeres. También puede manifestarse en adultos mayores, aunque es infrecuente; al respecto, en la actualidad se han reportado aproximadamente diez casos en los últimos diez años (11). Uno de los más recientes fue publicado por la revista Respiratory Medicine en 2010, y en éste se expone el caso de un paciente de 50 años de edad, hospitalizado por insuficiencia respiratoria aguda, cuya biopsia mediante estudio anatomopatológico reveló hemosiderosis pulmonar. De otra parte, aunque puede haber remisiones espontáneas, el pronóstico a largo plazo es pobre debido a que la mayoría de los pacientes desarrollan fibrosis pulmonar dentro de los cinco años posteriores al inicio de su manifestación (12).

\section{Fisiopatología}

Aunque la patogenia de la hemosiderosis pulmonar idiopática sigue siendo desconocida, hoy se postulan varias teorías que intentan explicar la ruptura de la membrana alvéolo-capilar (13) las cuales se mencionan a continuación:

\section{Teoría autoinmune}

En algunos estudios en pacientes con hemosiderosis pulmonar idiopática se observaron, mediante microscopia electrónica (14), lesiones en la membrana alvéolocapilar. A nivel ultraestructural (15) se documentó vacuolización, engrosamiento de las células alveolares y endoteliales, así como ruptura de las membranas 
basales capilares alveolares. Estas primeras descripciones datan de épocas anteriores a la disponibilidad de ensayos inmunológicos (16).

Algún tiempo después, durante 23 años, se investigó una base autoinmune sobre la demostración de complejos inmunes circulantes en el plasma (17), aunque las pruebas de inmunohistoquímica de tejido pulmonar no han apoyado una patogenia inmunológica (18); curiosamente $25 \%$ de los pacientes que han sobrevivido más de diez años con hemosiderosis pulmonar idiopática, desarrollan alguna forma autoimune de la enfermedad (19).

\section{Teoría alérgica}

Se origina como una hipótesis de enfoque sistémico en consideración a que algunos pacientes con hemosiderosis pulmonar idiopática presentan anticuerpos lgE detectables en plasma, acompañados generalmente de otras patologías como enfermedad celíaca (20). Hasta la fecha se han reportado más de diez casos publicados en la literatura; incluso en dichos informes sugieren que los pacientes con hemosiderosis pulmonar idiopática, parecían tener una remisión después de la instauración de una dieta libre de gluten, si bien este hecho puede corresponder a una asociación causal (21).

\section{Teoría inflamatoria}

Factores de origen ambiental o intrínseco pueden dar lugar al depósito de células inflamatorias, como los neutrófilos que al acumularse en la membrana alvéolocapilar causan su disrupción. Algunos autores proponen una asociación entre la exposición a insecticidas, toxinas de hongos, específicamente Stachybotrys chartarum, que pueden ser los agentes desencadenantes de dichas reacciones (22).

\section{Teoría metabólica}

El defecto en el metabolismo del hierro ha sido propuesto como causa de hemosiderosis pulmonar idiopática. El hierro constituye un elemento esencial en el metabolismo de todos los seres vivos; es el segundo metal más abundante de la tierra, está presente en grandes cantidades en la corteza terrestre, y a pesar de ello su deficiencia nutricional es cada día más frecuente. La fuente de hierro a los pulmones se da mediante la circulación, donde se encuentra unido a la transferrina o lactoferrina, así como también la inhalación (humo de cigarrillo, polvo metálico) o desde el metabolismo del eritrocito como ocurre en la hemosiderosis pulmonar idiopática (23).

Dentro de los macrófagos, el hierro se elimina de la hemoglobina mediante una reacción enzimática catalizada por la enzima hemo-oxigenasa 1, que puede ser inducida por factores de estrés celular, incluyendo diversas citocinas. Aunque las vías intracelulares del metabolismo del hierro dentro del macrófago no se entienden claramente, parece que hay una capacidad limitada de los macrófagos alveolares de metabolizar la hemoglobina y en algunos casos esto puede estar relacionado con la falta de hemo-oxigenasa. En general, la importancia de comprender la ferrocinética se relaciona con el hecho de que la capacidad de los macrófagos alveolares para metabolizar el hierro, es fácilmente agotable, y que la presencia de hierro libre en los alvéolos puede provocar lesiones locales y fibrosis (23).

\section{Manifestaciones clínicas}

Clásicamente se ha descrito una tríada caracterizada por anemia, hemoptisis e inflitrados pulmonares, signos secundarios al sangrado intra-alveolar difuso. Sin embargo, pueden darse diversos tipos de presentaciones clínicas $(22,23)$.

En la fase aguda, por ejemplo, existen síntomas y signos inespecíficos, dados por fiebre, cefalea, tos, taquipnea, dolor abdominal, hepatoesplenomegalia transitoria; y en etapas más progresivas, crisis de hemoptisis de diferente cuantía que en algunas ocasiones pone en riesgo la vida (24).

Existe una presentación insidiosa que cursa con anemia ferropriva progresiva refractaria a la suplementación con hierro; además se presentan síntomas respiratorios recurrentes crónicos, tos, disnea, cianosis, sibilancias, crépitos y episodios recurrentes de sangrado (25).

\section{Lupus eritematoso sistémico}

La hemorragia alveolar difusa es una manifestación infrecuente en los pacientes con lupus eritematoso sistémico; en algunas cohortes ha sido reportada entre $1 \%$ hasta 5,4\% (26). Puede aparecer en etapas tempranas o tardías de la enfermedad, y constituye un factor de mal pronóstico. Representa $1,5 \%$ a $3,7 \%$ de ingresos al hospital en los pacientes con lupus eritematoso sistémico, de ahí que se requiera tratamiento intensivo inmediato, y posee una tasa alta de mortalidad que va desde $23 \%$ hasta $92 \%$ (27). Informes recientes sugieren que el manejo agresivo con altas dosis de corticosteroides, ciclofosfamida y plasmaféresis aumenta la supervivencia de al menos el $60 \%$ (28).

Esta grave manifestación de la enfermedad debe ser parte del diagnóstico diferencial en todos los pacientes con lupus eritematoso sistémico que presentan hemoptisis e infiltrados pulmonares difusos y caída de la hemoglobina. En estos casos, para descartar infección 
y confirmar el diagnóstico, se indica la broncoscopia con lavado bronco-alveolar (BAL) (29). Las lesiones histopatológicas que acompañan la hemorragia alveolar en los pacientes con lupus eritematoso sistémico, incluyen vasculopatía trombógena con escasas reacciones inflamatorias, que agrupan anticuerpos contra las células endoteliales, inmunocomplejos depositados en el endotelio (depósitos de IgG y fracciones de complemento con aspecto granular dentro de las paredes alveolares) y otros factores que alteran la coagulación; también se pueden hallar vasculitis linfocítica, reacciones vaculares neutrofilícas y vasculitis sistémica (30).

Su reconocimiento temprano permitirá iniciar una terapia agresiva rápidamente, debido a que en estos pacientes la muerte ocurre por insuficiencia respiratoria y su instauración se da en cuestión de horas o días a la presentación inicial (31).

\section{Síndrome de Goodpasture}

El síndrome de Goodpasture es una enfermedad autoinmune rara, caracterizada por la tríada de hemorragia pulmonar, glomerulonefritis proliferativa y anticuerpos antimembrana basal glomerular (32). Sólo 5\% a 10\% se manifiestan como hemorragia alveolar difusa, y ocurre más en pacientes fumadores o que tienen exposición a hidrocarbonos volátiles. En $60 \%$ a $80 \%$ de los casos, existe enfermedad renal casi simultáneamente con enfermedad pulmonar; sólo 5\% a 10\% tiene presentación únicamente pulmonar. Este síndrome tiene una alta mortalidad que sobrepasa el $80 \%$ a seis meses, y de éstos una mitad fallece de hemorragia alveolar difusa y la otra de insuficiencia renal (33).

El síndrome de Goodpasture es considerado como un trastorno autoinmune contra el dominio 1 de la proteína no colágeno carboxiterminal (NC1) de la cadena alfa 3 del colágeno tipo IV, caracterizado por la presencia de anticuerpos contra la membrana basal alveolar y glomerular (34), con depósito de inmunoglobulinas, en especial IgG a lo largo de estas membranas, hecho que puede confirmarse mediante una biopsia pulmonar con inmunofluorescencia directa (35). La unión de los autoanticuerpos con la membrana basal, inicia una respuesta inflamatoria que cursa con quimiotaxis y liberación de radicales libres que puede generar una lesión tisular, y además expresarse como hemorragia pulmonar y glomerulonefritis proliferativa. Inicialmente, puede haber manifestaciones respiratorias como hemoptisis debido a la infiltración neutrofilíca con daño en la microvasculatura alveolar y después aparecerán las manifestaciones de glomerulonefritis (36).

\section{Lesión alveolar difusa}

El daño alveolar difuso es un tipo de enfermedad intersticial pulmonar y un modelo de lesión pulmonar aguda, en la cual hay una injuria extensa y severa de la unidad alvéolo - capilar. Se relaciona con pacientes que han sufrido insuficiencia respiratoria aguda del adulto en correlación con el hallazgo histológico (37). Su etiología es múltiple y pueden identificarse en la práctica clínica, antecedentes de trauma, sepsis, inhalación de productos tóxicos o drogas (38), los cuales pueden producir lesión pulmonar por dos vías patológicas: la primera, una vía directa en donde la injuria ingresa directamente a la unidad alvéolo-capilar (inhalación de tóxicos, contusión pulmonar, neumonía), y la segunda, que hace referencia a la injuria pulmonar producida por una respuesta sistémica (sepsis, síndrome de respuesta inflamatoria sistémica), con posterior manifestación de hemoptisis secundaria a hemorragia alveolar difusa (39).

\section{Toxicidad por penicilamina}

La hemorragia alveolar difusa secundaria a la toxicidad por fármacos como la penicilamina (producto de degradación de la penicilina, quelante de cobre, utilizada por primera vez en 1956 como tratamiento para la enfermedad de Wilson) (40), es controversial ya que la mayor parte de los casos se describe en pacientes con artritis reumatoide. Las lesiones pulmonares que se producen tanto por el fármaco como por la enfermedad, son muy similares y pueden crear confusión; en caso de toxicidad, existen algunos patrones histológicos orientativos como la hiperplasia linfoide, casi siempre sin vasculitis y la bronquiolitis obliterante; también se han reportando casos en la literatura en donde se presentan estos patrones histológicos en pacientes tratados con penicilamina para la enfermedad de Wilson, poniéndose de manifiesto la acción del fármaco $(30,38)$.

\section{Estenosis mitral}

Las manifestaciones de la enfermedad valvular mitral en el parénquima pulmonar, que ocurren en 7,7\% (41), son el resultado del incremento de la presión venosa pulmonar en pacientes con estenosis mitral. La patogenia se caracteriza por hemorragia alveolar intermitente, con posterior acumulación anormal de hierro (doscientas veces mayor a los valores normales). Cada episodio agudo de hemorragia pulmonar es seguido por un período de remisión variable, lo cual puede demostrarse mediante estudio con lavado bronco-alveolar (BAL); los hallazgos radiológicos incluyen cefalización del flujo pulmonar, edema intersticial, perivascular o perialveolar en algunas ocasiones, y hemorragia alveolar difusa (áreas acinares difusas, confluentes, con preservación del parénquima periférico) (42). 


\section{Enfermedad veno-oclusiva}

La hemorragia alveolar difusa puede ser la primera manifestación de enfermedad veno-oclusiva periférica e incluso de enfermedades del tejido conectivo (43). En estos casos el curso de la enfermedad usualmente tiene un mal pronóstico (44). De otro lado, se han reportado series de casos de enfermedad veno-oclusiva pulmonar como forma infrecuente de hipertensión arterial pulmonar en aproximadamente $10 \%$ de los casos, comúnmente en niños y adultos jóvenes (45), que conduce a elevación de la resistencia vascular pulmonar, genera una lesión endotelial como evento inicial desencadenante y finalmente puede producir hemorragia alveolar difusa oculta que podrá ser evidenciada mediante un estudio con lavado bronco-alveolar (BAL) (46).

\section{Hemangiomatosis}

La hemangiomatosis capilar pulmonar es un trastorno anormal caracterizado por la proliferación de capilares alveolares que infiltran el parénquima pulmonar, particularmente bronquíolos, vénulas e intersticio, y que clínicamente se hace pasar por hipertensión arterial idiopática $(47,48)$. Además, existe hemorragia alveolar difusa, que clínicamente cursa con disnea progresiva, hemoptisis, y ocasionalmente dolor pleurítico e insuficiencia respiratoria que en algunos casos puede ser letal (49).

\section{Linfangioleiomiomatosis/Esclerosis tuberosa}

Es una enfermedad rara que afecta particularmente a mujeres en edad fértil, con carácter multisistémico. Se puede presentar de forma esporádica o en asociación con la esclerosis tuberosa (TSC). Se caracteriza por una proliferación anormal de células musculares lisas inmaduras que cursan con disfunción de los genes TSC1 y TSC2, encargados de codificar hamartina y tuberina respectivamente, dando lugar a la activación y movilidad de células lisas inmaduras (50), con crecimiento aberrante sobre la vía aérea, el parénquima pulmonar, vasos linfáticos y vasos sanguíneos pulmonares que pueden generar hemorragia alveolar difusa, con evolución progresiva hacia la insuficiencia respiratoria aguda (51); este último factor de muy mal pronóstico (52). La aparición de la linfangioleiomiomatosis esencialmente en su totalidad, ocurre en mujeres en edad fértil, pues concuerda con la hipótesis de que los agentes anti-estrógenos podrían prevenir la progresión de la enfermedad, como por ejemplo los análogos de la hormona liberadora de gonadotrofinas (53).

\section{CONCLUSIÓN}

El paciente con hemorragia alveolar difusa constituye un reto para el profesional en salud, aunque su pre- sentación es infrecuente; puede aparecer en múltiples enfermedades, incluso secundaria a complicaciones o estadios avanzados de diferentes patologías, incluir insuficiencia respiratoria aguda y llevar hasta la muerte, si no se instaura un manejo apropiado (54).

En resumen, las hemorragias alveolares difusas son emergencias médicas y pueden generar finales catastróficos de no diagnosticarlas y tratarlas a tiempo. La clave diagnóstica se basa en una historia clínica detallada, una exploración física adecuada y el apoyo en ayudas paraclínicas, que orientarán finalmente a la etiología más probable (30).

\section{BIBLIOGRAFÍA}

1. Specks U. Diffuse alveolar hemorrhage syndromes. Current Opinion in Rheumatology 2001; 13 (1): 12-17.

2. loachimescu OC, Stoller JK. Diffuse alveolar hemorrhage: diagnosing it and finding the cause. Cleve Clin J Med 2008; 75 (4): 258: $260-265$.

3. Lara AR, Schwarz MI. Diffuse alveolar hemorrhage. Chest 2010; 137 (5): 1164-7.

4. Newsome, Brandi R. Diffuse alveolar hemorrhage. Southern Medical Journal 2011; 104 (4): 269-274.

5. McCabe C, Jones Q, Nikolopoulou A. Pulmonary-renal syndromes: An update for respiratory physicians. Respir Med 2011 Jun 17. [Epub ahead of print].

6. Jara LJ, Vera-Lastra O, Calleja MC. Pulmonary-renal vasculitic disorders: differential diagnosis and management. Curr Rheumatol Rep 2003; 5 (2): 107-15.

7. Hellmich B, Gross WL. Difficult to diagnose manifestations of vasculitis: does an interdisciplinary approach help? Best Pract Res Clin Rheumatol 2005; 19 (2): 243-61.

8. Cordier JF, Cottin V. Alveolar hemorrhage in vasculitis: primary and secondary. Semin Respir Crit Care Med 2011; 32 (3): 310-21.

9. Willms H, Gutjahr K. Diagnostics and therapy of idiopathic pulmonary hemosiderosis. Med Klin (Munich) 2007; 102 (6): 445-50.

10. Oviedo Ramírez MI, Hop K, Carrera E, Soriano Rosas J. Idiopathic pulmonary haemosiderosis in a young adult. Autopsy findings Arch Bronconeumol 2010; 46 (10): 565-7. Epub 2010.Aug 13.

11. Chen $\mathrm{CH}$, Yang HB, Chiang SR, Wang PC. Idiopathic pulmonary hemosiderosis: favorable response to corticosteroids. J Chin Med Assoc 2008; 71 (8): 421-4.

12. Chermiti BA Fatma, Chtourou A, Mahouchi R. Idiopathic pulmonary hemosiderosis in adult. Respiratory Medicine CME 2010; 3 (4): 238-240.

13. Ioachimescu O. Idiopathic pulmonary hemosiderosis in adults. Clinical Pulmonary Medicine 2005; 12 (1): 16-25.

14. Dolan CJ Jr, Srodes CH, Duffy FD. Idiopathic pulmonary hemosiderosis: electron microscopic, immunofluorescent, and iron kinetic studies. Chest 1975; 68: 577-580.

15. Corrin B, Jagusch $M$, Dewar $A$, et al. Fine structural changes in idiopathic pulmonary haemosiderosis. J Pathol 1987; 153: 249-256.

16. Irwin RS, Cottrell TS, Hsu KC, et al. Idiopathic pulmonary hemosiderosis: an electron microscopic and immunofluorescent study. Chest 1974; 65: 41-45. 
17. Blanco A, Solis P, Gómez S, et al. C1q-binding immune complexes and other immunological studies in children with pulmonary hemosiderosis. Allergol Immunopathol (Madr) 1984; 12: 37-44.

18. Fullmer JJ, Langston C. Pulmonary capillaritis in children: a review of eight cases with comparison to other alveolar hemorrhage syndromes The Journal of Pediatrics 2005; 146 (3): 376-38.

19. Le Clainche L, Le Bourgeois M, Fauroux B, et al. Long-term outcome of idiopathic pulmonary hemosiderosis in children. Medicine (Baltimore). 2000; 79: 318-326.

20. Mayes DH, Guerrero ML A few good men: a Marine with hemoptysis and diarrhea. Idiopathic pulmonary hemosiderosis and celiac sprue. Chest 2008; 134 (3): 644-7.

21. Khemiri M, Ouederni M. Screening for celiac disease in idiopathic pulmonary hemosiderosis. Gastroenterol Clin Biol 2008; 32 (8-9): 745-8

22. Centers for Disease Control and Prevention. Update: pulmonary hemorrhage/hemosiderosis among infants: Cleveland, Ohio, 1993-1996. JAMA 2000; 283: 1951-1953.

23. Rao NR, Goodman LR. Smoking-related interstitial lung disease. Annals of Diagnostic Pathology 2008; 12 (6): 445-457.

24. Deniz O, Ongürü O. Idiopathic pulmonary hemosiderosis in an adult patient responded well to corticosteroid therapy. Tuberk Toraks 2007; 55 (1): 77-82.

25. Nuesslein TG, Teig N. Pulmonary haemosiderosis in infants and children. Paediatric Respiratory Reviews 2006; 7 (1): 45-48.

26. Olser W. On the visceral manifestations of the erythema group of skin diseases. Am J Med Sci 2009; 338 (5): 396-408.

27. Santos Ocampo AS, Mandell BF, Fessler BJ. Alveolar hemorrhage in systemic lupus erythematosus: presentation and management. Chest 2000; 118 (4): 1083-90.

28. Badsha $\mathrm{H}$, et al. Pulmonary hemorrhage in systemic lupus erythematosus. Seminars in Arthritis and Rheumatism 2004; 33 (6): 414-42.

29. Pego-Reigosa JM, Medeiros DA. Respiratory manifestations of systemic lupus erythematosus: old and new concepts. Best Practice \& Research Clinical Rheumatology 2009; 23 (4): 469-480.

30. Gómez-Román JJ. Diffuse alveolar hemorrhage. Archivos de Bronconeumología (English Edition). 2008; 44 (8): 428-436.

31. Mahmoud GA, Gheith RE. Alveolar hemorrhage in systemic lupus erythematosus: An overview. The Egyptian Rheumatologist 2011; 33 (1): 1-1.

32. O'Hara, Collin, Song, Joo Young. Goodpasture's syndrome with negative serology. Pathology Case Reviews 2007; 12 (3): 109-112.

33. Brandi R. Diffuse alveolar hemorrhage. Southern Medical Journal 2011; 104 (4): 269-274.

34. Lê Quang C, Delèvaux I. Intra-alveolar haemorrhage without renal damage as the initial presenting feature of Goodpasture's syndrome: case report and review of literature. Rev Med Interne 2008; 29 (12): 1038-42. Epub 2008 Jun 24.
35. Cui Z, Zhao J, Jia XY. Clinical features and outcomes of antiglomerular basement membrane disease in older patients. Am J Kidney Dis 2011; 57 (4): 575-82. Epub 2010 Dec 18.

36. Shah MK, Hugghins SY. Characteristics and outcomes of patients with Goodpasture's syndrome. South Med J 2002; 95 (12): 1411-8.

37. Tomashefski JF Jr. Pulmonary pathology of acute respiratory distress syndrome. Clin Chest Med 2000; 21 (3): 435-466.

38. Huppmann AR. Drug-induced lung disease. Pathology Case Reviews 2007; 12 (3): 113-117.

39. Castro CY. ARDS and diffuse alveolar damage: a pathologist's perspective. Semin Thorac Cardiovasc Surg 2006; 18 (1): 13-9.

40. Case JP. Old and new drugs used in rheumatoid arthritis: a historical perspective. Part 1: the older drugs. Am J Ther 2001; 8 (2): 123-43.

41. Ishida K, Seki R. Clinical aspect of diffuse alveolar hemorrhage syndrome. Nihon Kokyuki Gakkai Zasshi 2003; 41 (12): 851-6.

42. Cohen S. Diffuse pulmonary hemorrhage: evolutionary 'flaw' or consequence of evolutionary progress? Am J Med Sci 2002; 323 (3): 130-9.

43. O'Callaghan DS, Dorfmuller P. Pulmonary veno-occlusive disease: the bête noire of pulmonary hypertension in connective tissue diseases? Presse Med 2011; 40 (1 Pt 2): e65-78. Epub 2011 Jan 5.

44. Barboza CE, Jardim CV. Pulmonary veno-occlusive disease: diagnostic and therapeutic alternatives. J Bras Pneumol 2008; 34 (9): 749-52.

45. Lantuéjoul S, Sheppard MN. Pulmonary veno-occlusive disease and pulmonary capillary hemangiomatosis: a clinicopathologic study of 35 cases. Am J Surg Pathol 2006; 30 (7): 850-7.

46. Montani D, O'Callaghan DS, Pulmonary veno-occlusive disease: recent progress and current challenges. Respir Med 2010; 104 (Suppl. 1): S23-32. Epub 2010 Apr 24.

47. Sullivan A, Chmura K Pulmonary capillary hemangiomatosis: an immunohistochemical analysis of vascular remodeling. Eur J Med Res 2006; 11 (5): 187-93.

48. Almagro P, Julià J. Pulmonary capillary hemangiomatosis associated with primary pulmonary hypertension: report of 2 new cases and review of 35 cases from the literatura. Medicine (Baltimore) 2002; 81 (6): 417-24.

49. Kothari S. Pulmonary capillary hemangiomatosis. Circulation 2009; 120 (4): 352-354.

50. Pacheco-Rodríguez G, Moss J. The role of chemokines in migration of metastatic-like lymphangioleiomyomatosis cells. Crit Rev Immunol 2010; 30 (4): 387-94.

51. Ansótegui Barrera E, Mancheño Franch N. Lymphangioleiomyomatosis. Archivos de Bronconeumología (English Edition) 2011; 47 (2): 85-93.

52. Gao J, Zhu P. A clinicopathological analysis of pulmonary lymphangioleiomyomatosis. Zhongguo Fei Ai Za Zhi 2011; 14 (4): 378-82.

53. Glasgow CG, Steagall WK. Lymphangioleiomyomatosis (LAM): molecular insights lead to targeted therapies. Respir Med 2010; 104 (Suppl. 1): S45-58.

54. Díaz J, Calamia KT, Lee AS. Pulmonary vasculitis in the intensive care unit. J Intensive Care Med 2011; 26 (2): 88-104. 